\title{
Nearly exact mining of frequent trees in large networks
}

\author{
Ashraf M. Kibriya · Jan Ramon
}

Received: 18 November 2012 / Accepted: 6 May 2013

(C) The Author(s) 2013

\begin{abstract}
Mining frequent patterns in a single network (graph) poses a number of challenges. Already only to match one path pattern to a network under subgraph isomorphism is NP-complete. Classical matching algorithms become intractable even for reasonably small patterns, on networks which are large or have a high average degree. Based on recent advances in parameterized complexity theory, we propose a novel miner for rooted trees in networks. The miner, for a fixed parameter $k$ (maximal pattern size), can mine all rooted trees with delay linear in the size of the network and only mildly exponential in the fixed parameter $k$. This allows us to mine tractably, rooted trees, in large networks such as the WWW or social networks. We establish the practical applicability of our miner, by presenting an experimental evaluation on both synthetic and real-world data.
\end{abstract}

Keywords Graph mining · Pattern mining · Pattern matching · Isomorphism • Subgraph isomorphism $\cdot$ Homomorphism

\section{Introduction}

Mining frequent patterns is one of the fundamental tasks of data mining. Traditionally, patterns have consisted of simple sets of items. However, since the last decade interest has been building up in mining more structured forms of patterns, such as trees and

Responsible editors: Peter Flach, Tijl De Bie and Nello Cristianini

\footnotetext{
A. M. Kibriya $(\varangle) \cdot$ J. Ramon

Department of Computer Science, KU Leuven, Leuven, Belgium

e-mail: ashraf.kibriya@cs.kuleuven.be

J. Ramon

e-mail: jan.ramon@cs.kuleuven.be
} 
arbitrary graphs. This has especially been the case due to the phenomenal growth of structured data sources such as the WWW, and social and citation networks.

There are generally two settings for mining in graphs. The first setting is the transactional one, where we are given a set of graphs and a threshold $t$, and we want to find patterns that occur in at least $t$ graphs in the set. The second graph mining setting, which we will consider in this paper, is the single network setting, where we are given a single graph and a threshold $t$, and we want to find patterns that have a support of at least $t$ in the given single graph according to some appropriate support measure.

Central to any pattern mining task is the notion of pattern matching. In graph mining, subgraph isomorphism is usually the matching operator of choice. Checking for even a simple path in a graph under subgraph isomorphism is well known to be NP-complete. Indeed, the problem of finding a path in a graph can be reduced to the Hamiltonian path problem. However, recent advances in the theory of parameterized complexity have produced algorithms that can tractably solve several of the computationally hard graph problems, including subgraph isomorphism, when certain parameters of the problems are bounded. The work of Koutis and Williams (2009) is particularly relevant in this case. It gives a randomized algorithm for deciding subgraph isomorphism of a tree in a network with an asymptotic complexity of $O\left(k^{2} \log ^{2}(k) m 2^{k}\right)$, where $m$ is the number of edges in the network and $k$ the size of the pattern.

In this paper we build on the work of Koutis and Williams (2009). We present an algorithm to mine all frequent rooted tree patterns with delay $O\left(k^{2} \log ^{2}(k) m 2^{k}\right)$, i.e. the time between any two consecutive frequent patterns being output is bounded by $O\left(k^{2} \log ^{2}(k) m 2^{k}\right)$. We present an implementation and experiments on both synthetic and real-world data. To the best of our knowledge, our work is the first to tractably mine tree patterns under subgraph isomorphism in single large (possibly dense) networks.

This work is an extension of our earlier work presented in Kibriya and Ramon (2012). Here, we provide a more detailed description of our optimizations, the work of Koutis and Williams (2009) and complexity theory. We add material describing frequency sampling and potential applications of our miner. We also present extended experimental results, and include additional figures and supporting text for better clarity of the theory.

The rest of the paper is structured as follows. In the next section we give a brief overview of the related work. In Sect. 3 we review some of the preliminaries required for explaining our work. In Sect. 4, we formally define the problem we are tackling, and then proceed and present the details of our work in Sect. 5, while also reviewing the work of Koutis and Williams (2009). In Sect. 6, we present a thorough experimental evaluation of our miner to establish its practical applicability, followed by a discussion on some of its potential applications in Sect. 7. We then conclude with some final remarks and future direction of our research in Sect. 8.

\section{Related work}

Most of the work done so far in graph mining is in the transactional setting. For example, graph miners AGM (Inokuchi et al. 2000), FSG (Kuramochi, Karypis 2001), FFSM (Huan et al. 2003), gSpan (Yan and Han 2002), MoFa/MoSS (Borgelt and 
Berthold 2002; Borgelt et al. 2005), FOG (Horváth et al. 2010) and Gaston (Nijssen and Kok 2004), are all designed for the transactional setting. At a high level, all these methods get as input a database $D$ of graphs and a minimal frequency threshold $t$, and produce as output the set of all patterns $P$ for which $P$ occurs in at least $t$ graphs in the database $D$.

These miners employ a variety of optimizations to reduce the overall runtime and memory use, including canonical forms to avoid duplicate generation of candidates and suitable subgraph isomorphism tests. The earlier generation of miners, AGM and FSG, work in an apriori style fashion, whereas others, e.g. FFSM and Gaston, use depth first search to optimize memory use (Wörlein et al. 2005). Furthermore, as in itemset mining, considerable work has also been done in pattern summarization, by mining more representative and comprehensive graph patterns such as closed graphs (Yan and Han 2003; Chi et al. 2005) and maximal graphs (Huan et al. 2004; Thomas et al. 2010). For transactional graph mining systems, the computational cost of pattern matching is high but often manageable, as often the transaction graphs are small, or the structure of the these graphs can be exploited (Horváth and Ramon 2010; Horváth et al. 2010; Borgelt et al. 2005).

In addition to the above there are also some transactional miners proposed specifically for trees (Chi et al. 2004; Chehreghani 2011). But they only work for mining trees in trees, not, as in our case, mining trees in general/arbitrary graphs.

For mining in single networks, work has so far been limited. The most notable ones are by Kuramochi and Karypis (2005), Jiang et al. (2009) and the SUBDUE system (Cook and Holder 1994, 2000). The one by Kuramochi and Karypis (2005), is a general purpose algorithm for mining arbitrary graph patterns in networks, but is only efficient for sparse networks and relatively small patterns. This also holds for the more recent (Jiang et al. 2009) which is only demonstrated for less dense networks (networks with vertex degree $\leq 25$ ). SUBDUE is one of the earliest systems proposed for finding patterns in a single network. Its task is different however, as it does not exhaustively search for patterns. It is an approximate algorithm that uses heuristic search to find patterns that can best compress the network (based on the minimum description length principle). Even though approximate, it does not scale well to large networks even if the networks are sparse (Kuramochi and Karypis 2005).

Other works that exist for single networks are for mining evolution of networks (Berlingerio et al. 2009; Bogdanov et al. 2011), node/edge classification (Rossi et al. 2012; Henderson et al. 2011; Gallagher et al. 2008), or mining for patterns using homomorphism as the matching operator (Dries and Nijssen 2012). Even though Berlingerio et al. (2009) mine for patterns when mining their evolution rules, the language of the patterns is specific to time evolving networks, and excludes more general (unlabeled) patterns like trees or cycles.

For pattern matching, with subgraph isomorphism as the matching operator, usually the miners use one of a number of base matching algorithms. For general arbitrary graphs, Ullman (1976) and VF2 (Cordella et al. 2001) are often the popular choices. Both of them are branch-and-bound based algorithms that employ backtracking and pruning strategies to eliminate large parts of the search space. VF2 is newer and in comparison provides mostly better runtime than Ullman (Cordella et al. 2004). 
An alternative to subgraph isomorphism is to use homomorphism. Homomorphism, as used in Dries and Nijssen (2012), has a lower computational cost (only polynomial in the network size), but has other disadvantages. One of the major reasons why interest in mining conjunctive queries has been declining is that candidate generation is problematic, as illustrated by the fact that no optimal refinement operator exists (Nienhuys-Cheng and De Wolf 1997; Nijssen and Kok, personal communication). Moreover, depending on the application, requiring pattern vertices to map to different network vertices (as in isomorphism) may be the most natural choice. For instance, consider a family relationship graph. Suppose that we want to construct a pattern matching everyone having two brothers. With subgraph isomorphism, one can just make a pattern consisting of a person node and a node for each brother, and an edge labeled brother between the person node and each of the brother nodes. With homomorphism, such can not be specified so easily. A solution may be to define a relation "is not equal" but such relation would make the graph very dense.

\section{Preliminaries}

\subsection{Graph theory}

We recall basic graph theoretic notions used in this paper. For more background in this area, we refer the reader to Diestel (2010).

Definition 1 (Graph) A graph is a pair $G=\left(V_{G}, E_{G}\right)$, where $V_{G} \neq \emptyset$ is a finite set of vertices/nodes, and $E_{G} \subseteq\left\{\{x, y\} \mid x, y \in V_{G}\right\}$ a set of edges connecting those vertices. For any graph $G$ its vertex and edge sets are also referred to as $V(G)$ and $E(G)$ respectively. If $\{u, v\} \in E(G)$, we say $u$ and $v$ are adjacent vertices and the edge $\{u, v\}$ is incident with the vertex $v$. In this paper, we call $|V(G)|$ the size of $G$.

Definition 2 (Labeled Graph) A labeled graph is a quadruple $G=\left(V_{G}, E_{G}, \Sigma_{G}, \lambda_{G}\right)$, where $\left(V_{G}, E_{G}\right)$ is a graph, $\Sigma_{G} \neq \emptyset$ a set of labels, and $\lambda_{G}: V_{G} \cup E_{G} \rightarrow \Sigma_{G}$ a function assigning labels to vertices and edges.

Definition 3 (Path, tree and rooted tree) A path in a graph $G$ is a sequence $\left\{v_{1}, v_{2}, \ldots, v_{n}\right\}$ of pairwise distinct vertices of $G$ such that:

$$
\left\{v_{i}, v_{i+1}\right\} \in E(G) \text {, for all } 1 \leq i<n .
$$

A tree is a graph such that there is a unique path between any pair of its vertices.

A rooted tree is a tree $T$ in which a single vertex $r \in V(T)$, denoted by $\operatorname{root}(T)$, is distinguished and is called the root. One can represent a rooted tree and its root with a directed graph where all edges are directed away from the root.

Definition 4 (Subgraph, (iso/homo)-morphism \& graph embedding) A graph $H=\left(V_{H}, E_{H}\right)$ is called a subgraph of $G=\left(V_{G}, E_{G}\right)$, if $V_{H} \subseteq V_{G}$ and $E_{H} \subseteq E_{G}$. It is an induced subgraph if

$$
\forall v, v^{\prime} \in V_{H},\left\{v, v^{\prime}\right\} \in E_{H} \Leftrightarrow\left\{v, v^{\prime}\right\} \in E_{G} .
$$


Otherwise it is a non-induced subgraph.

A graph $H=\left(V_{H}, E_{H}\right)$ is said to be isomorphic to $G=\left(V_{G}, E_{G}\right)$ (denoted by $H \cong G$ ), if there exists an edge-preserving bijective mapping of $H$ onto $G$. For labeled graphs the mapping, in addition to edge-preserving, also has to be label-preserving. Formally, $H \cong G$ if there exists a function $\varphi: V_{H} \rightarrow V_{G}$ such that:

$$
\forall u, v \in V_{H},\{u, v\} \in E_{H} \Leftrightarrow\{\varphi(u), \varphi(v)\} \in E_{G},
$$

and for the labeled case additionally,

$$
\forall u \in V_{H}, \lambda_{H}(u)=\lambda_{G}(\varphi(u)),
$$

and

$$
\forall\{u, v\} \in E_{H}, \lambda_{H}(\{u, v\})=\lambda_{G}(\{\varphi(u), \varphi(v)\}) .
$$

If $H$ is isomorphic to a subgraph of $G$, then we call $H$ subgraph isomorphic to $G$ and write $H \preceq G$. In that case, the mapping is called an embedding of $H$ in $G$.

If a mapping from $H$ to $G$ is edge-preserving but not bijective (and hence not one-to-one), then it defines a homomorphism between $H$ and $G$.

We denote with $\operatorname{Emb}(H, G)$, the set of all isomorphic embeddings of $H$ in $G$. Note, that (i) the number of embeddings $|\operatorname{Emb}(H, G)|$ can be exponential, and that (ii) in this paper we consider normal subgraph isomorphism rather than the more restrictive induced subgraph isomorphism.

\subsection{Group theory}

In this section, we briefly review some basic concepts on group theory. These will be mainly used in Sect. 5.2, when describing the Koutis-Williams algorithm.

Definition 5 (Group) A group $G$ is a set of elements endowed with an arbitrary binary operation $(\cdot)$, such that it satisfies the following four properties, known as the group axioms:

- Closure If $a, b \in G$, then so does $a \cdot b$.

- Associativity $(a \cdot b) \cdot c=a \cdot(b \cdot c)$.

- Identity There exists an element $i \in G$, such that for every $a \in G: a \cdot i=i \cdot a=a$.

- Inverse For every $a \in G$, there exists an element $a^{-1}$, such that $a \cdot a^{-1}=i$

Definition 6 (Ring) A ring is a set of elements with two specified binary operations, addition $(+)$ and multiplication $(x)$. It must satisfy all the group axioms for $(+)$, all but the inverse axiom for $(\times)$, and the following additional axioms:

- Commutativity of (+) For all $a, b \in G, a+b=b+a$.

- Distributivity of ( $\times)$ over (+) For all $a, b, c \in G, a \times(b+c)=a \times b+a \times c$ and $(a+b) \times c=a \times c+b \times c$. 
If in addition to the above if it also satisfies commutativity of $(x)$, then it is called a commutative ring, otherwise a non-commutative ring.

Definition 7 (Field) A field is a commutative ring for which also the inverse axiom for $\times$ holds.

For a ring R, an integer $n$ and $a \in R, n . a=\sum_{i=1}^{n} a$ is called the scalar multiplication between $n$ and $a$. For any finite field $F$, it is necessarily the case that there exists an integer $n>0$, such that for every $a \in F, n a=0$. The smallest such $n$ for a field is called its characteristic.

\subsection{Parameterized complexity}

Parameterized complexity theory deals with fine grained analysis of problems that are computationally hard. It is based on the observation that many computationally hard problems have some inherent property that depends on some parameter $k$, given as part of the input. The basic idea is to push the exponential dependence of problems to this parameter $k$. Hence, for fixed values of $k$ there is a polynomial algorithm. In practice, if $k$ is small with respect to the rest of the input, then problems for which all known algorithms are exponential in $k$ may become tractable. E.g., for the VERTEX COVER problem (VERTEX COVER gets as input a graph $G=(V, E)$ and asks to find a subset $C \subseteq V$ such that all edges $e \in E$ have at least one of their endpoints in $C)$, the best known non-parameterized solution is a $O\left(1.211^{n(=|V|)}\right)$ time algorithm (Robson 1986), whereas a parameterized solution (if we know that a solution $C$ exists with $|C| \leq k)$ gives us a $O\left(k n+1.271^{k}\right)$ time algorithm (Chen et al. 2001). Thus if $k \leq 0.79 n$ the parameterized solution would run faster. In many practical applications it is often the case that $k \ll n$, and hence a parameterized solution would often be a better choice. In the context of the current paper, we are considering subgraph isomorphism between patterns, whose number of vertices $k$ is typically at most a few dozen, and large networks whose number of vertices $n$ ranges typically in the millions (possibly even billions). Deciding subgraph isomorphism is NP-complete, but the parameters $n$ and $k$ differ orders of magnitude. Hence, for this specific application, a parameterized algorithm only exponential in $k$ is an interesting option.

Below are some definitions and related concepts often used in the field.

Definition 8 (Parameterized problem) A parameterized problem is a subset $\Pi$ $\in \Sigma^{*} \times \mathbb{N}$, where $\Sigma$ is some alphabet (also known as a parameterized language since a subset of $\Sigma^{*}$ is a language over alphabet $\Sigma$ ). Input for a parameterized problem is of the form $(I, k) \in \Sigma^{*} \times \mathbb{N}$, where $I$ is called the main part and $k$ the parameter of the input.

Definition 9 (Fixed parameter tractability) A parameterized problem $\Pi$ is fixed parameter tractable (FPT), if there is a function $f: \mathbb{N} \rightarrow \mathbb{N}$ and an algorithm that decides whether an input $(I, k) \in \Pi$ in $O\left(f(k) . n^{c}\right)$ steps (where $c$ is some constant independent of $k$ ). The problem is said to belong to the class FPT, and the algorithm is called an FPT-algorithm. If no such algorithm exists then the problem is fixed parameter intractable. 
Definition 10 ( $O^{*}$ Notation) Often the complexity of FPT algorithms are expressed in the $O^{*}$ notation. $O^{*}$ notation simply suppresses all the polynomial parts and shows only the exponential factors of the normal $O$ notation. So, for e.g., $O\left(k n+1.271^{k}\right)$ is $O^{*}\left(1.271^{k}\right)$, and $O\left(1.211^{n}\right)$ is $O^{*}\left(1.211^{n}\right)$.

\section{Problem statement}

Let $\mathcal{G}$ be the language of all graphs, let $\mathcal{L}_{p} \subseteq \mathcal{G}$ be a language of patterns, let $M: \mathcal{L}_{P} \times \mathcal{G} \rightarrow \mathbb{R}$ be some measure of interestingness, let $t$ be a given threshold of interestingness and $G \in \mathcal{G}$ be the network we want to mine patterns in. Then, we would like to compute the set $\mathcal{F}_{\left(\mathcal{L}_{p}, G, M, t\right)}$ of interesting patterns defined by:

$$
\mathcal{F}_{\left(\mathcal{L}_{p}, G, M, t\right)}=\left\{T \in \mathcal{L}_{P}: M(T, G) \geq t\right\}
$$

In our case, our pattern language is the class of rooted trees.

Several frequency measures have been proposed (as measures of interestingness) in the literature for single graph mining (Bringmann and Nijssen 2007; Calders et al. 2011; Wang and Ramon 2012). This paper primarily focuses on the matching of patterns, and though our methods are general, for simplicity we restrict ourselves to the frequency measure obtained by counting the number of possible images of the root of a rooted tree pattern. This measure is anti-monotone w.r.t. increasing pattern size, and is defined as follows.

Definition 11 (Root image) The root image of a rooted tree $T$ in $G$ is the set of all vertices $v \in G$ to which $\operatorname{root}(T)$ can be mapped under subgraph isomorphism, i.e.,

$$
\mathcal{R I}(T, G)=\{\varphi(\operatorname{root}(T)) \mid \varphi \in \operatorname{Emb}(T, G)\},
$$

Definition 12 (Support) Let $T$ be a rooted tree and $G$ be a graph. Then, we define the support of $T$ in $G$ as the size of its root image, i.e.,

$$
\operatorname{supp}(T, G)=|\mathcal{R} \mathcal{I}(T, G)| .
$$

Figure 1 shows an example of our above defined 'root-image' support measure. In the figure the root $r$ of our pattern can only be mapped to nodes $x_{1}, x_{2}$ and $x_{3}$, and not to $x_{4}$, under subgraph isomorphism and hence its frequency is 3 .

For the remainder of the paper, we will consider a network $G$ and for brevity we will use $n=|V(G)|$ and $m=|E(G)|$. Moreover, the symbol $T$ will be used to refer to a rooted tree pattern and we will denote its size with $k=|V(T)|$. We will abuse terminology and use 'tree' for 'rooted tree' if it is clear from the context.

\section{Mining frequent rooted trees}

In order to realize a pattern miner for rooted trees in single networks, the two key ingredients are the generation of candidate trees and the efficient counting of the frequency 
Fig. 1 Support measure based on root images. Frequency of $P$ in $G$ is 3

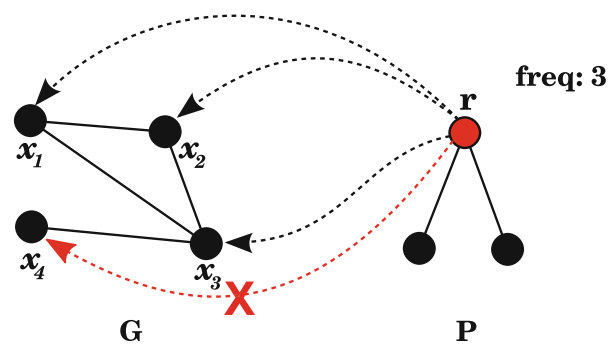

of patterns in the network (using subgraph isomorphism). We, therefore, first proceed by outlining our candidate generation method. Then, we review the subgraph isomorphism method of Koutis and Williams (2009), which we will call Koutis-Williams method from here on, and show how their method can be employed to compute our above defined root-image frequency measure. We then give the complexity bounds of our complete miner, and then outline some of the high-level optimizations that we implemented for our miner. Finally, we give a discussion on sampling the frequency instead of counting it exactly.

\subsection{Candidate generation}

In our miner, we use the same technique for generating candidate rooted trees as by Nijssen and Kok (2004) and Nijssen and Kok (2005). It generates rooted ordered trees that are left heavy, i.e. children of a node are ordered and each left sub-tree is larger than the right sub-tree according to their canonical form. The left heavy property avoids generating trees that are isomorphically equivalent.

The method works by adding nodes only to the right most path, and ensuring that the condition of left heaviness is met with each new added node. The method thus produces a new tree for each added node, and can do so with delay $O(k)$ for size $k$ trees. The left heavy subtree condition is met by maintaining a canonical form for the trees. The canonical form is defined as the string of the form $C(T)=\left(\operatorname{depth}\left(v_{1}\right), \operatorname{depth}\left(v_{2}\right), \cdots, \operatorname{depth}\left(v_{k}\right)\right)$ which is lexicographically heaviest among all trees of size $k$. The sequence $\left(\operatorname{depth}\left(v_{1}\right), \operatorname{depth}\left(v_{2}\right), \cdots, \operatorname{depth}\left(v_{k}\right)\right)$ is formed by doing a pre-order traversal of the tree. By maintaining such canonical strings, a new node can only be inserted in a tree at positions where the left substring is lexicographically larger than the right substring after insertion. If the trees are labeled, then vertex and edge labels are also inserted together with the depth sequence. Figure 2 gives some example trees, both labeled and unlabeled, with their corresponding canonical string to illustrate the technique.

5.2 Subgraph isomorphism and frequency counting with the Koutis-Williams algorithm

The Koutis-Williams method exploits the fact that for trees, subgraph homomorphism can be decided in polynomial time. The method essentially consists of two core parts. In the first part, it constructs an arithmetic circuit computing a polynomial $P$ representing 


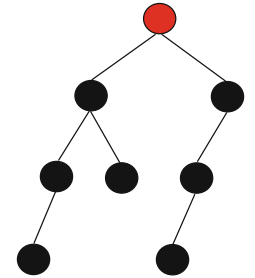

$(0,1,2,3,2,1,2,3)$

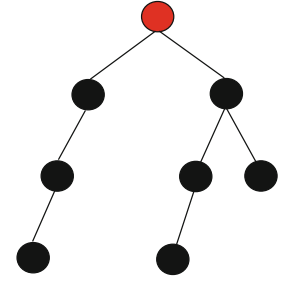

$(0,1,2,3,1,2,3,2)$

(b)

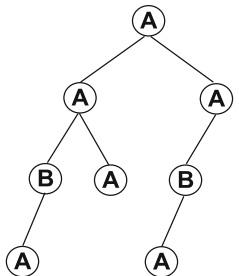

$\{(0, A)(1, A)(2, B)(3, A)(2, A)(1, A)(2, B)(3, A)\}$

(c)

Fig. 2 The (rooted) unlabeled trees $\mathbf{a}$ and $\mathbf{b}$ are isomorphic to each other, but $\mathbf{a}$ is left-heavy compared to b. We can treat as canonical form, the lexicographically heaviest string of pre-order traversal of depth sequence (given below each tree), and generate only trees that are like a. Figure c shows an example of a left-heavy labeled tree with its corresponding canonical string

all possible homomorphisms of a tree $T$ in a network. In particular, with every network vertex $v$ a variable $x_{v}$ is associated, and every homomorphism $\pi$ from the pattern $T$ to the network $G$ corresponds to a term (monomial) $\prod_{v \in V(T)} x_{\pi(v)}$, i.e. the product of variables corresponding to the mapping of the vertices of the pattern. A multilinear term is a term where every variable occurs with degree at most 1 , so e.g. $x_{u} x_{v} x_{w}$ is a multilinear term, but $x_{u} x_{v}^{2}$ is not. Isomorphisms are injective, and therefore the terms in $P$ corresponding to isomorphisms will be exactly the multilinear terms of $P$. Figure $3 \mathrm{a}$ and $\mathrm{b}$ gives an illustration of this concept. Figure $3 \mathrm{a}$ shows the mapping of tree $T$ onto a network $G$, and the corresponding polynomial representing all possible homomorphisms of $T$ in $G$, if the mapping of root $t_{1} \in V(T)$ is fixed to $x_{1} \in V(G)$. If we fix this mapping, then we have two options for $t_{2}$ and $t_{3}$ : (i) we map both $t_{1}$ and $t_{2}$ to one vertex, giving us terms $x_{1} x_{2}^{2}$ and $x_{1} x_{3}^{2}$, and (ii) we map one of them to $x_{2}$ and the other to $x_{3}$, giving us two multilinear terms $x_{1} x_{2} x_{3}$ and $x_{1} x_{3} x_{2}$ in the polynomial. The last two multilinear terms are the ones that represent isomorphisms, while, the homomorphisms corresponding to the non-multilinear terms $x_{1} x_{2}^{2}$ and $x_{1} x_{3}^{2}$ are not isomorphisms. As stated earlier, the polynomial gets constructed in the form of an arithmetic circuit. An arithmetic circuit is a condensed representation of a polynomial. While the number of terms in the polynomial may be of the order $O\left(n^{k}\right)$, the number of gates in the arithmetic circuit is only $O(m k)$. Figure $3 \mathrm{~b}$ shows the arithmetic circuit of the polynomial in Fig. 3a. The circuit has a node $C_{T_{r}, j}$ for every subtree $T_{r}$ of the pattern $T$ and every vertex $j$ of the network $G$. The circuit is created based on the following recursion relation:

$$
C_{T_{r}, j}:= \begin{cases}x_{j}, & \text { if }\left|V\left(T_{r}\right)\right|=1 \\ \prod_{r^{\prime}:\left(r, r^{\prime}\right) \in E\left(T_{r}\right)}\left(\sum_{j^{\prime}:\left(j, j^{\prime}\right) \in E(G)} x_{j} . C_{T_{r^{\prime}}, j^{\prime}}\right), & \text { otherwise }\end{cases}
$$

where $T_{r}$ is tree with root $r$ and $T_{r^{\prime}} \subset T_{r}$ is its subtree, rooted at $r^{\prime}$. The relation essentially allows to create a series of product and sum gates defining a circuit, similar to Fig. 3b, representing a polynomial of all possible homomorphisms of $T_{r}$ in $G$ with mapping of $r \in V\left(T_{r}\right)$ fixed to $j \in V(G)$. The algorithm thus, is a dynamic 


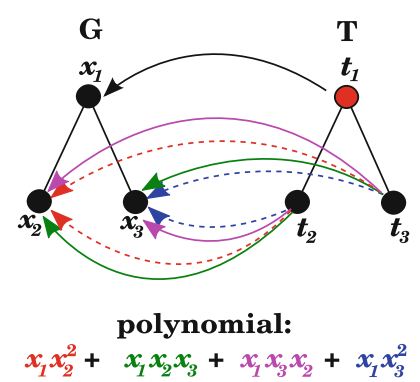

(a)

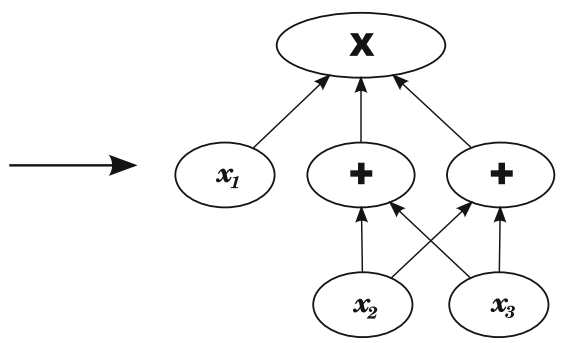

Arithmetic circuit for: $x_{1}\left\{\left(x_{2}+x_{3}\right)\left(x_{2}+x_{3}\right)\right\}$

(b)

Fig. 3 All homomorphisms of $T$ which map the root $t_{1}$ on $x_{1}$. Solid lines represent mappings that are also isomorphic

programming style method where each internal (sum or product) gate needs to be visited only once, and can be implemented using a simple table.

In its second part, the Koutis-Williams method then evaluates the polynomial. The polynomial $P$ is evaluated on an appropriate commutative group algebra, that ensures all terms with squares evaluate to zero. Therefore, all terms which are not multilinear (i.e. all homomorphisms which are not isomorphisms; in the example above all terms except the multilinear terms $x_{1} x_{2} x_{3}$ and $\left.x_{1} x_{3} x_{2}\right)$ vanish. As detailed later, the multilinear terms may or may not evaluate to non-zero depending on the randomization of the algorithm. As a result, if there exists a subgraph isomorphism between $T$ and $G$, evaluating $P$ may give a non-zero result, as we will see later with probability at least 0.2 , while if there is no such subgraph isomorphism, we are sure $P$ will evaluate to zero. Repeating this evaluation a sufficient number of times with independent randomizations can boost this probability so that the risk that we make a mistake gets arbitrarily small. In Fig. 3 for simplicity of explanation we fixed the mapping of root $t_{1}$ to $x_{1}$. This corresponds to using the arithmetic circuit to decide whether there is a subgraph isomorphism between $T$ and $G$ mapping $t_{1}$ on $x_{1}$. We can extend this circuit to contain output gates for each of the possible images of the root $t_{1}$ (i.e. mapping of $t_{1}$ to each of remaining $x_{2}, x_{3}$ and $x_{4}$ ) to let it compute everything needed to calculate the full count of the above defined root-image support measure. An example for the labeled case is shown in Fig. 4. The recursion relation for circuit creation, similar to the one given above, is outlined in Algorithm 1 (note, in case of unmatching labels we simply multiply a term by zero thereby avoid creating subcircuit for parts of the network whose labels do not match).

In particular, in Koutis and Williams (2009), the polynomial $P$ is evaluated over $G F\left(2^{l}\right) \mathbb{Z}_{2}^{k}$. This is an algebraic structure built up of simpler ingredients. Let us review these structures from the beginning. First, $\mathbb{Z}_{2}$ is the field with two possible values 0 and 1 , where addition is interpreted as XOR. Its operation tables are shown in Table 1. $\mathbb{Z}_{2}^{k}$ is a commutative group consisting of all bitvectors of length $k$. For $x \in \mathbb{Z}_{2}^{k}$, we denote with $x_{i}$ its $i$ th component. The group operation, $\cdot$ is defined as component-wise addition modulo 2 of the elements of the bit vectors, i.e. for all $x, y \in \mathbb{Z}_{2}^{k}$,

$$
(x \cdot y)_{i}=x_{i}+y_{i}
$$


Fig. 4 All homomorphisms of labeled $T$ in network $G$, with mapping of root $t_{1}$ fixed to $x_{1}$

Table 1 Operations in $\mathbb{Z}_{2}$

Fig. 5 Example computation in $\mathbb{Z}_{2}^{k},+$

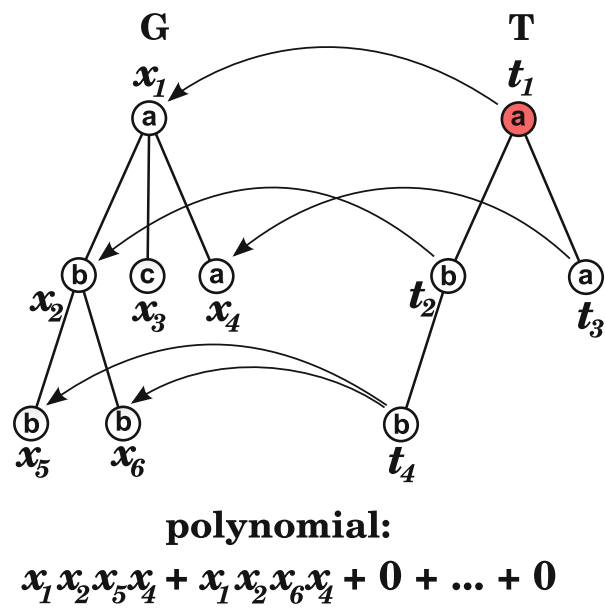

Table 2 Operations in $G F\left(2^{2}\right)$

\begin{tabular}{|c|c|c|c|c|c|c|c|c|c|}
\hline$G F\left(2^{2}\right),+$ & 0 & 1 & $\mathrm{x}$ & $\mathrm{x}+1$ & $G F\left(2^{2}\right)$, & 0 & 1 & $\mathrm{x}$ & $x+1$ \\
\hline 0 & 0 & 1 & $\mathrm{x}$ & $x+1$ & 0 & 0 & 0 & 0 & 0 \\
\hline 1 & 1 & 0 & $x+1$ & $\mathrm{x}$ & 1 & 0 & 1 & $\mathrm{x}$ & $x+1$ \\
\hline $\mathrm{x}$ & $\mathrm{x}$ & $x+1$ & 0 & 1 & $\mathrm{x}$ & 0 & $\mathrm{x}$ & $\mathrm{x}+1$ & 1 \\
\hline$x+1$ & $\mathrm{x}+1$ & $\mathrm{x}$ & 1 & 0 & $x+1$ & 0 & $x+1$ & 1 & $\mathrm{x}$ \\
\hline
\end{tabular}

where the + is the addition operation in $\mathbb{Z}_{2}$. Please note that the group operation of $\mathbb{Z}_{2}^{k}$ is denoted $\cdot$ rather than + here (Fig. 5). The neutral or the identity element of $\mathbb{Z}_{2}^{k}$ is the zero vector, denoted by $W_{0}$, containing $k$ zeros.

$G F\left(2^{l}\right)$ is the unique field with $2^{l}$ elements. Its elements are most commonly represented with binary polynomials modulo an irreducible binary polynomial. As an example, Table 2 gives the operation tables for $G F\left(2^{2}\right)$ represented with binary polynomials of degree at most 1 modulo $x^{2}+x+1$. Please note that $G F\left(2^{l}\right)$ has characteristic 2, i.e. $a+a=0$ holds for any $a \in G F\left(2^{l}\right)$.

Finally, $G F\left(2^{l}\right) \mathbb{Z}_{2}^{k}$ is a ring, the set of all linear combinations of elements of $\mathbb{Z}_{2}^{k}$ with coefficients from $G F\left(2^{l}\right)$. In particular, for $a \in G F\left(2^{l}\right) \mathbb{Z}_{2}^{k}$, there is a coefficient 


$$
\begin{gathered}
\left(x\left[\begin{array}{l}
1 \\
1 \\
0
\end{array}\right]+(x+1)\left[\begin{array}{l}
0 \\
1 \\
1
\end{array}\right]\right)+\left(x\left[\begin{array}{l}
0 \\
1 \\
1
\end{array}\right]+\left[\begin{array}{l}
0 \\
1 \\
0
\end{array}\right]\right)=x\left[\begin{array}{l}
1 \\
1 \\
0
\end{array}\right]+\left[\begin{array}{l}
0 \\
1 \\
1
\end{array}\right]+\left[\begin{array}{l}
0 \\
1 \\
0
\end{array}\right] \\
\left(x\left[\begin{array}{l}
1 \\
1 \\
0
\end{array}\right]+(x+1)\left[\begin{array}{l}
0 \\
1 \\
1
\end{array}\right]\right)\left(x\left[\begin{array}{l}
0 \\
1 \\
1
\end{array}\right]+\left[\begin{array}{l}
0 \\
1 \\
0
\end{array}\right]\right)=(x+1)\left[\begin{array}{l}
1 \\
0 \\
1
\end{array}\right]+\left[\begin{array}{l}
0 \\
0 \\
0
\end{array}\right]+x\left[\begin{array}{l}
1 \\
0 \\
0
\end{array}\right]+(x+1)\left[\begin{array}{l}
0 \\
0 \\
1
\end{array}\right]
\end{gathered}
$$

Fig. 6 Example calculation in $G F\left(2^{2}\right) \mathbb{Z}_{2}^{k}$

$a_{g}$ for each $g \in \mathbb{Z}_{2}^{k}$ such that $a=\sum_{g \in \mathbb{Z}_{2}^{k}} a_{g} g$. If $a=\sum_{g \in \mathbb{Z}_{2}^{k}} a_{g} g$ and $b=\sum_{g \in \mathbb{Z}_{2}^{k}} b_{g} g$ then

$$
a+b=\sum_{g \in \mathbb{Z}_{2}^{k}} a_{g} g+\sum_{g \in \mathbb{Z}_{2}^{k}} b_{g} g=\sum_{g \in \mathbb{Z}_{2}^{k}}\left(a_{g}+b_{g}\right) g
$$

and

$$
a \cdot b=\left(\sum_{g \in \mathbb{Z}_{2}^{k}} a_{g} g\right) \cdot\left(\sum_{g \in \mathbb{Z}_{2}^{k}} b_{g} g\right)=\sum_{g \in \mathbb{Z}_{2}^{k}}\left(\sum_{h \in \mathbb{Z}_{2}^{k}} a_{h} b_{g} \cdot h\right) g
$$

Figure 6 gives some example calculations.

An important property of the aforementioned ring is that for every $g \in \mathbb{Z}_{2}^{k},\left(W_{0}+\right.$ $g)^{2}=0$. This is easily seen by writing $\left(W_{0}+g\right)^{2}=W_{0}^{2}+W_{0} \cdot g+g \cdot W_{0}+g \cdot g$ and by noting that $g \cdot g=W_{0}$ and $W_{0} \cdot g=g$ for all $g$; after which $g+g=0$ and $W_{0}+W_{0}=0$ due to the characteristic 2 of $G F\left(2^{l}\right)$. For instance,

$$
\begin{aligned}
\left(W_{0}+\left[\begin{array}{l}
1 \\
0 \\
1
\end{array}\right]\right)^{2} & =\left(\left[\begin{array}{l}
0 \\
0 \\
0
\end{array}\right]+\left[\begin{array}{l}
1 \\
0 \\
1
\end{array}\right]\right)^{2} \\
& =\left[\begin{array}{l}
0 \\
0 \\
0
\end{array}\right]\left[\begin{array}{l}
0 \\
0 \\
0
\end{array}\right]+\left[\begin{array}{l}
0 \\
0 \\
0
\end{array}\right]\left[\begin{array}{l}
1 \\
0 \\
1
\end{array}\right]+\left[\begin{array}{l}
1 \\
0 \\
1
\end{array}\right]\left[\begin{array}{l}
0 \\
0 \\
0
\end{array}\right]+\left[\begin{array}{l}
1 \\
0 \\
1
\end{array}\right]\left[\begin{array}{l}
1 \\
0 \\
1
\end{array}\right] \\
& =\left[\begin{array}{l}
0 \\
0 \\
0
\end{array}\right]+\left[\begin{array}{l}
1 \\
0 \\
1
\end{array}\right]+\left[\begin{array}{l}
1 \\
0 \\
1
\end{array}\right]+\left[\begin{array}{l}
0 \\
0 \\
0
\end{array}\right]=2\left[\begin{array}{l}
0 \\
0 \\
0
\end{array}\right]+2\left[\begin{array}{l}
1 \\
0 \\
1
\end{array}\right]=0
\end{aligned}
$$

This is the crucial property which ensures that all non-multilinear terms in the polynomial $P$ evaluate to zero.

So let us now return to the evaluation of the polynomial $P$ representing all homomorphisms from $T$ to $G$. For evaluation, each $x_{v}$ in the polynomial or each $x_{v}$ node in the circuit (called an input-gate) is substituted with $\left(W_{0}+y_{v}\right)$, where $y_{v}$ is drawn randomly from $\mathbb{Z}_{2}^{k} \backslash\left\{W_{0}\right\}$. Furthermore, the output of each product-gate is multiplied by a random coefficient drawn from $G F\left(2^{l}\right) \backslash\{0\}$. The arithmetic is performed over $G F\left(2^{l}\right) \mathbb{Z}_{2}^{k}$ throughout the circuit. One can show that the randomization of values for the $x_{v}$ 's ensures that multilinear terms evaluate to non-zero with probability at 
least $1 / 4$. Moreover, with $l$ sufficiently large, the randomization of the coefficients after the product gates ${ }^{1}$ ensures that the summation of non-zero terms evaluates to non-zero with probability at least 7/8. The result of Koutis and Williams (2009) is based on the following observations:

- For a set $S \subseteq V(G)$ and variables $x_{v}=W_{0}+y_{v}$ with $y_{v} \in \mathbb{Z}_{2}^{k}$, it holds that $\prod_{v \in S} x_{v} \neq 0$ iff the multiset $\left\{y_{v} \mid v \in S\right\}$ is a set of linearly independent vectors (non-multilinear terms therefore always evaluate to 0 ).

- A set of $k$ randomly chosen bitvectors of length $k$ is linearly independent with probability at least $1 / 4$ (as the probability of a random $k \times k$ matrix over a finite field to have full rank is at least $1 / 4$ (Blum and Kannan 1995))

- For any set of elements $b_{i} \in G F\left(2^{l}\right) \mathbb{Z}_{2}^{k}$ and randomly chosen coefficients $a_{i} \in$ $G F\left(2^{l}\right)$, with $l=3+\log _{2} k$, if any of the $b_{i}$ is non-zero, then $\sum_{i} a_{i} b_{i}$ is zero with probability at most $k /|F|=k / 2^{3+\log _{2} k}=1 / 8$ (due to the Schwartz-Zippel Lemma (Motwani and Raghavan 1995) for degree- $k$ polynomials), and therefore it is non-zero with probability at least $7 / 8$.

The method by Koutis and Williams (2009), thus gives a non-zero evaluation of the circuit iff there is a multilinear term in the circuit, with probability at least $1 / 5$ $(>1 / 4 \cdot 7 / 8)$.

In Algorithm 1, we outline our implementation of the Koutis-Williams subgraph isomorphism method. The occur method in Algorithm 1 defines an arithmetic circuit of a polynomial for all homomorphic mappings with the mapping of $\operatorname{root} r \in T$ fixed to some $v^{\prime} \in G$. The creation and evaluation of such circuits for all $v^{\prime} \in G$, in method countFreq, gives us the full count of our above defined root-image support measure for our tree pattern $T$.

The $a_{r, j}$ and $x_{j}$, in the occur method, are chosen randomly from $G F\left(2^{l}\right)$ and $\mathbb{Z}_{2}^{k}$, and the arithmetic on the elements of array $C_{i, j}$ is performed over $G F\left(2^{l}\right) \mathbb{Z}_{2}^{k}$. In our implementation we use the representation-theoretic technique similar to Koutis (2008) for doing the evaluations, that is memory linear in $k$ (rather than linear in $\left.2^{k}\right)$. In particular, this method allows us to compute the coefficient in $G F\left(2^{l}\right)$ of each bitvector in $\mathbb{Z}_{2}^{k}$ separately, so that we do not need to propagate data structures of size $2^{k}$ through the arithmetic circuit but can rather propagate $2^{k}$ times elements from $G F\left(2^{l}\right)$ (in practice, it is sufficient to only evaluate the coefficient of $W_{0}$ ). The details of this technique are out of the scope of this article.

As per Koutis and Williams (2009), the theoretical space complexity of occur method is $O(\mathrm{~km})$ and its time complexity is $O\left(k^{2} m 2^{k} l^{2}\right){ }^{2}$ By extension the time complexity of countFreq method would be $O\left(k^{2} m n 2^{k} l^{2}\right)$. However, we note that since we use a dynamic programming style method, it is possible that with only a single evaluation of the arithmetic circuit to obtain the values occur $(\boldsymbol{T}, \boldsymbol{G}, \boldsymbol{r}, \boldsymbol{j})$ for all $j$ and all $r \in\{V(T) \backslash \operatorname{root}(T)\}$, and hence the time complexity is still only $O\left(k^{2} m 2^{k} l^{2}\right)$.

\footnotetext{
1 In our implementation however, we add random coefficients to the output of each input-gate. The two strategies are equivalent.

$2 O(k m)$ being the size of the circuit and $O\left(k 2^{k} l^{2}\right)$ to do arithmetic over $G F\left(2^{l}\right)\left[\mathbb{Z}_{2}^{k}\right]$
} 
As indicated earlier, the occur method's success probability $p>1 / 5$ can be increased to an arbitrary $p^{\prime}$, by repeating the method $\left\lceil\log \left(1-p^{\prime}\right) / \log (1-p)\right\rceil$ times (thereby decreasing the probability of failure).

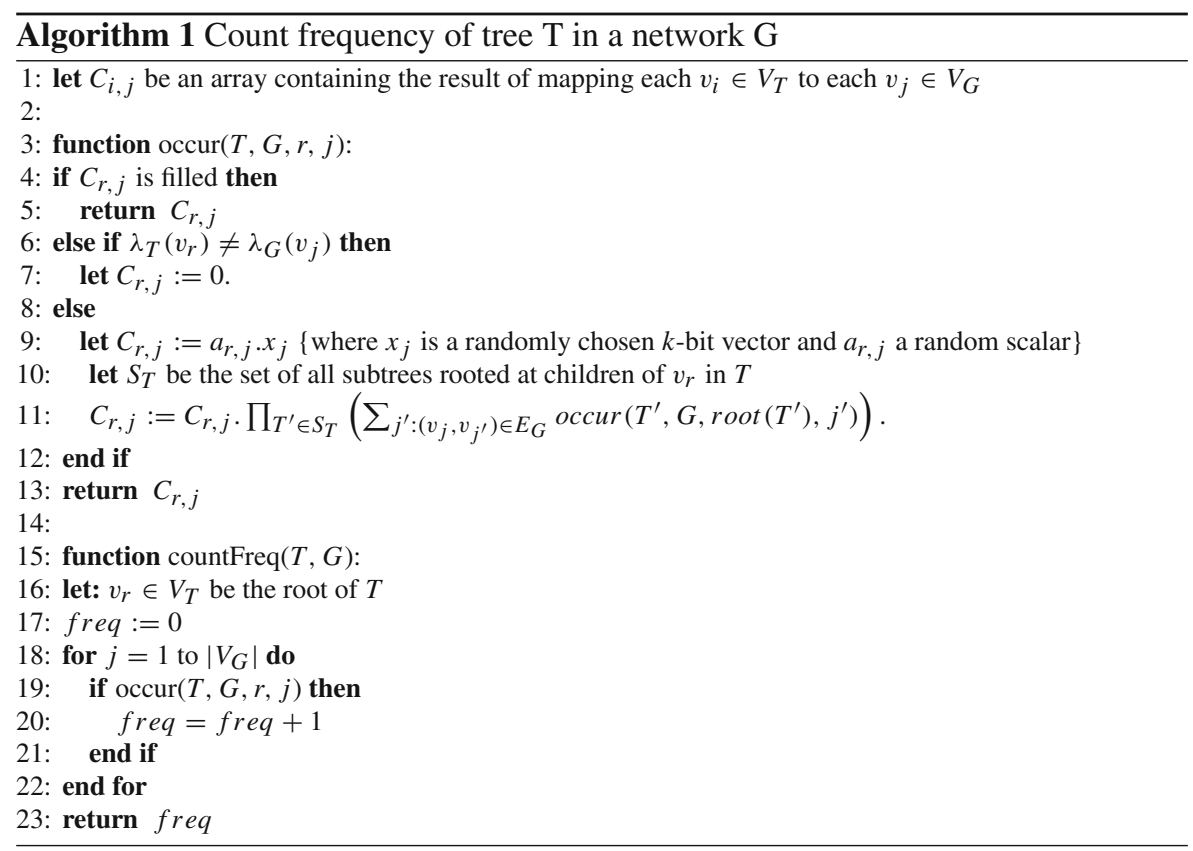

\subsection{Complete miner and complexity bounds}

Algorithm 2 gives pseudo-code for our complete pattern miner. It brings together all the core components to make an apriori style miner.

We now proceed to prove its theoretical bounds. Note, the space complexity of our miner is bounded by our main data structure $C_{i, j}$, and is $O(k n)$.

Theorem 1 Given a network $G$ with $n$ nodes and $m$ edges labeled by label set $\Sigma_{G}$, and a frequency threshold $t$ we can mine all frequent tree patterns of size $\leq k$ with delay $O\left(\left|\Sigma_{G}\right| \log ^{2}(k) k^{2} m 2^{k}\right)$.

Proof As mentioned earlier, the countFreq method takes $O\left(\log ^{2}(k) k m 2^{k}\right)$ time for each candidate tested. At each call made at level $i \geq 2$ the generateCandidates function in Algorithm 2 produces at most $O\left(\left|\Sigma_{G}\right| . k\right)$ candidates per frequent pattern in $S_{i-1}$. For each such candidate, countFreq is called, taking time $O\left(\log ^{2}(k) k m 2^{k}\right)$. Therefore, for each new solution output, the algorithm will need $O\left(\left|\Sigma_{G}\right| \log ^{2}(k) k^{2} m 2^{k}\right)$ more time to finish. The theorem follows by noting that we can delay the printing of the solutions (frequent patterns) in such a way that between the printing of each pair of consecutive solutions the delay is bounded by $O\left(\left|\Sigma_{G}\right| \log ^{2}(k) k^{2} m 2^{k}\right)$. 


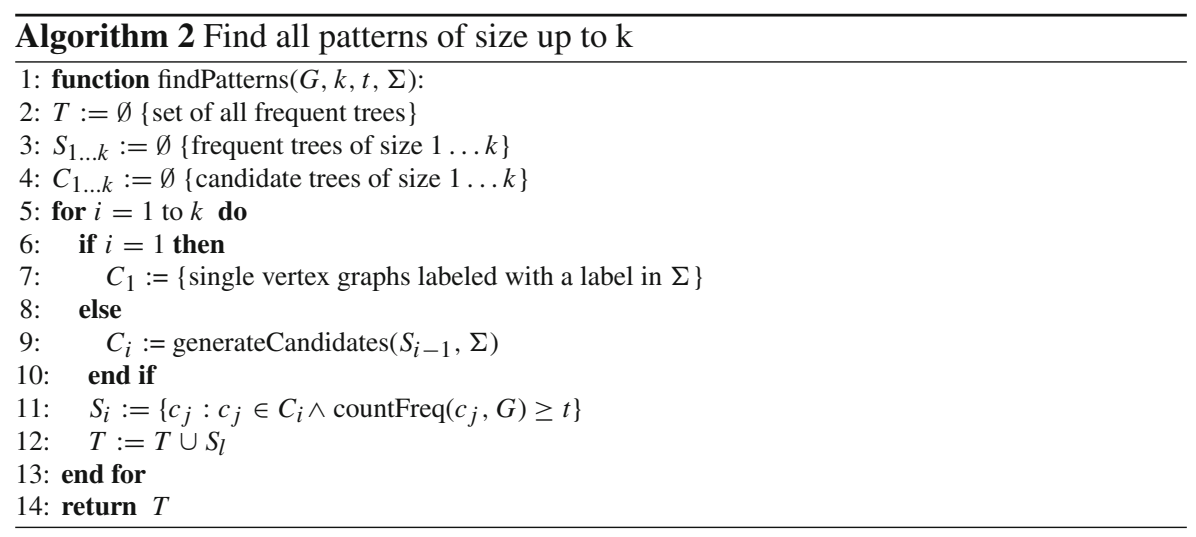

In particular, the algorithm works level-by-level and hence outputs its solutions in bursts, but at any time a sufficient number of solutions are known to ensure that the running time divided by the number of solutions found up to now is at most $O\left(\left|\Sigma_{G}\right| \log ^{2}(k) k^{2} m 2^{k}\right)$.

\subsection{Further optimizations}

A number of optimizations are still possible with Algorithm 2. Below we mention some of the more high level optimizations we implemented in our miner.

- Checking for homomorphisms Any subgraph isomorphism is also a homomorphism. Hence, if we can quickly check that there is no homomorphism between $T$ and $G$ (mapping the root of $T$ to a particular vertex of $G$ ), then we can eliminate the work of checking for subgraph isomorphism. As mentioned earlier, homomorphisms for trees in networks can be found in polynomial time. In fact in our case all we have to do is evaluate our circuit over integers, instead of the ring $G F\left(2^{l}\right)\left[\mathbb{Z}_{2}^{k}\right]$, thereby avoiding all the expensive arithmetic. Evaluation is linear in the size of the circuit and is only $O(\mathrm{~km})$. Note that we can perform this test not only for full trees but also for its subtrees (i.e. calculate and store $C_{r, j}$ for each $r \in\{V(T) \backslash \operatorname{root}(T)\}$ in Algorithm 1). At the expense of this linear amount of extra memory and time, we can thus store and use results of these inexpensive tests, and avoid the more expensive isomorphism tests for any part of the network and (sub)trees that do not match under homomorphism. As we see in our results, for labeled networks especially, this can offer considerable speedup.

- Sharing common subtrees Note that for each $C_{i}$ for $i \geq 2$ (in Algorithm 2), the candidate trees may share a number of subtrees common among them. We do not need to test each candidate tree in isolation. Instead we can reuse the previously computed results of the common subparts.

In our implementation we made a directed acyclic graph (DAG) structure without duplicate edges to represent all $T \in C_{i}$. Each node in this DAG represents either a root of a $T \in C_{i}$ or root of one of its subtrees. Several nodes can be parents of 
the same node and hence the corresponding trees can share subtrees. Note, that subtrees repeated in a single candidate $T$ are also repeated in the DAG (hence no duplicate edges), distinction between repeated subtrees in a single $T$ is needed to ensure our homomorphic mappings preserve bijections at circuit creation time (the occur method in Algorithm 1). This method shares computation at the expense of extra memory.

- Vertex label sort list As mentioned in the previous section, the space requirement of our miner is bounded trivially by our main data structure $C_{r, j}$ as $O(k n)$. We can, however, reduce this further for labeled networks. Instead of having a static $k \times n$ array, we can use a variable length array, and instead of reserving $n$ cells for each $k$, we reserve only $j_{\lambda_{T}(k)} \leq n$ cells, where $j_{\lambda_{T}(k)}=\mid\{v \in$ $\left.V(G): \lambda_{G}(v)=\lambda_{T}(k)\right\} \mid$ (i.e. the number of nodes in network having the same label as the $k$ th tree node). This offers considerable savings especially when combined with the above memory intensive optimization of sharing common subtrees.

To realize this variable length array, we create a vertex count list of length $\left|\Sigma_{G}\right|$, and a $O(n)$ vertex sort list. We count how many vertices belong to each $l \in \Sigma_{G}$, and while counting assign a new index to each network vertex in our vertex sort list. Our main data structure is then created based on the counts in the vertex count list, and its second dimension is accessed using the vertex sort list. The count and sort lists are created (and initialized) only once for a network, and can be used for all candidates trees generated and tested subsequently.

\subsection{Sampling the frequency}

Our algorithm is randomized but it can be used to obtain results which are correct with a very high confidence. In some cases, we may be only interested in collecting the frequent patterns and not necessarily in their exact frequency. In this section we discuss what we can gain by only approximating frequency. Our results show that, due to various circumstances, it may be hard to combine all our other optimizations with a sampling strategy.

There are two natural strategies which one could consider. First, one could evaluate only part of the arithmetic circuit and in that way check for only part of the network vertices whether they are the image of the pattern root under a subgraph isomorphism. Second, instead of running the algorithm until one is very confident that for every vertex which is a root image we will have observed a witness, we could run the algorithm only a few times.

Let us start with the first idea. suppose we check for only part of the network vertices whether they are the image of the pattern root under a subgraph isomorphism. When using a 'vanilla' (but space-expensive) implementation of the KoutisWilliams algorithm, this works decently. Unfortunately, there are two drawbacks. First, when sampling only a fraction $q$ of the candidate root images, we must compute a fraction larger than $q$ of the arithmetic circuit. The fraction of the arithmetic circuit we must compute increases quickly with increasing pattern size. Second, our 
implementation is a memory-efficient one, evaluating subgraph isomorphism by doing $2^{k}$ passes over the arithmetic circuit and only using linear space rather than the vanilla version which does one pass over the arithmetic circuit and requires exponential space. Therefore, if we calculate only part of the circuit and then conclude that our sample was not large enough to draw a definite conclusion w.r.t. the pattern being frequent or not, we can not resume computation and evaluate the mapping of the pattern root to the network vertices which were not in the original sample. Instead, we need to do (most of) the computations again. In summary, it is hard to give guarantees on the gain achieved when sampling only part of the root images.

The second idea one can pursue is to run the Koutis-Williams algorithm a smaller number of times, thereby lowering the confidence of its correctness for the individual potential root images. Let us first better analyze the randomized behavior of the KoutisWilliams algorithm. Suppose we run the Koutis-Williams algorithm once, evaluating for one randomization for all network vertices whether they are an image of the pattern root under an isomorphism. Then, what can we conclude from the result? We expect that a fraction of at least 0.2 of the root images will be found on average. However, the outcome for the several root images is not independent. E.g. suppose that our pattern has three vertices, i.e. one root and two leaves. Suppose that the two leaves have specific labels and only match to two specifically labeled vertices in the network. The frequency of the pattern is then the number of vertices having edges to both specially labeled network vertices. However, if the two vectors assigned to these two network vertices are linearly dependent, the arithmetic circuit will evaluate 0 for all potential root images. In conclusion, we can not really trust to find a fraction 0.2 (or any lower fraction) of the root images with one run of the Koutis-Williams algorithm. How about the other direction? We are sure that with probability at least 0.72 every multilinear term in the polynomial represented by the arithmetic circuit evaluates to zero. However, here again, we cannot trust that the real frequency is at least $1 /(1-0.72)$ times the number of successfully found root images in the first round. In particular, in practice many network vertices to which the pattern root can be mapped, will be the image of the pattern root under several different subgraph isomorphisms. Therefore, the probability that the Koutis-Williams algorithm will detect them as root image is significantly larger than 0.20 .

This last aspect also has a positive side: as in practice network vertices are often the image of the pattern root under several different isomorphisms, in many cases, a smaller number of iterations of the Koutis-Williams algorithm is typically necessary to detect all root images. Figure 7 shows for the DBLP network (described in the next section) what fraction of the root images were discovered after a given number of runs of the Koutis-Williams algorithm.

\section{Experimental evaluation}

Below we present an experimental evaluation of our miner on both synthetic and real-world data. Our main focus here is to show the efficacy of the method in practice. 


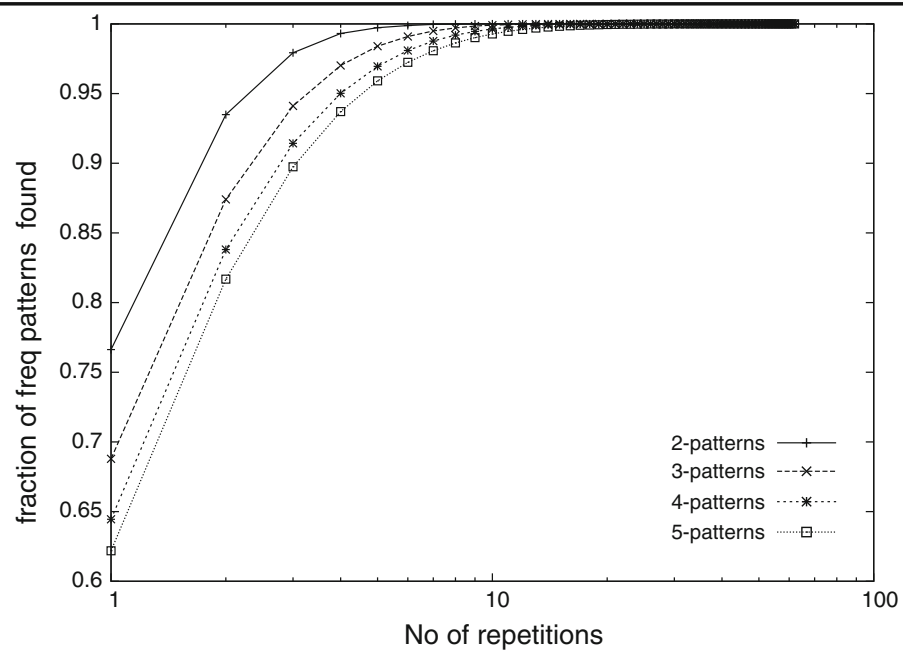

Fig. 7 The fraction of root images found as a function of the number of runs of the Koutis-Williams algorithm, when mining the DBLP network

\subsection{Experimental setup}

In our experimental evaluation, we are interested in the following experimental questions:

Q1 What size of patterns and networks can our algorithm handle within reasonable time?

Q2 How does our pattern matching strategy compare to state of the art strategies, in particular with VF2 (Cordella et al. 2001)?

Q3 Does our implementation scale as well as the theoretical Koutis-Williams algorithm?

Q4 What is the influence of pattern mining parameters and optimizations?

To perform our experiments, we implemented a system which we will call MINT (MIning Networks for Trees), containing a breadth-first pattern mining algorithm using the candidate generation method outlined in Sect. 5.1. We implemented the frequency counting based on the Koutis-Williams algorithm as described in Sect. 5.2, and a baseline MINT- VF2 using frequency counting based on the VF2 algorithm (Cordella et al. 2001). We consider several versions of our new algorithm: First, MINTSTD implements a vanilla version of the Koutis-Williams algorithm. Second, MINTHomo implements the Koutis-Williams method with the homomorphism checking optimization, and third, MINT- BATCH which includes homomorphism checking as well as sharing common subtrees among the candidates. We call the last one MiNTBATCH, as we share subtrees only among batchsize number candidates in each pass; otherwise the memory requirements get intractably large due to the exponential number of frequent patterns.

In order to be able to compare the randomized algorithm to the deterministic VF2, the subgraph isomorphism tests were repeated a sufficient number of times to achieve a very high probability of success $\left(1-10^{-6}\right)$. The result was that the output of our ran- 
domized algorithm only deviated from the deterministic once the number of frequent patterns grew larger than $10^{6}$, in which case our method missed some frequent patterns (recall our randomized algorithm has only one sided error, it never reports infrequent patterns as frequent).

\subsection{Data sets}

We present results on both synthetic as well as real-world data.

For synthetic data we generated networks of size $n=\left\{10^{2}, 10^{3}, 10^{4}, 10^{5}, 10^{6}, 10^{7}\right\}$, and then randomly assigned 1 of 4 labels to each of the vertices. We generated networks from power-law model with degree distribution $P(d) \propto d^{-4}$. Such graphs show significant clustering, as is often seen in real-world data.

For real-world data, we used the DBLP citation network ${ }^{3}$, the Facebook social network $^{4}$, and the IMDB movie database. ${ }^{5}$

The DBLP data is a snapshot of their citation network from 1992 to 2007. It is a time evolving network, where edges are labeled by the year an author collaborated with another author, and nodes (i.e. authors) are unlabeled. It is the same data as used by Berlingerio et al. (2009).

The Facebook data is the Facebook friendship network obtained through two random sampling strategies: one through uniform sampling, and the other through independent Metropolis-Hastings random walks (Gjoka et al. 2010). For each strategy, the raw data consists of two files: one is the actual friendship data, represented as an adjacency list, and the other contains additional properties such as total numbers of friends and privacy settings for each userid. We merged the two files into a single network. For the merge, we started with the friendship file and removed all userids that were not present in the properties file, then we labeled each node (userid) with its privacy setting, and used a default label (i.e. no label) for the edges.

For IMDB, we extracted the movie-actor network from the raw database. Our extracted network consists of movie, year, role and actor nodes. Movie and role nodes were labeled by movie and role type, whereas year nodes were labeled by the year the movie was released in. Actor nodes as well as all edges are left with a default label.

Table 3 gives basic statistics of all our real-world networks.

\subsection{Results}

Complete mining of synthetic data We ran the algorithms on synthetic datasets, and mined for as large patterns as we could, using frequency threshold of $\mathbf{0 . 1}$, for the duration of 1 week. Table 4 gives the number of frequent patterns found in that time period, as a function of the network size and pattern size. It is noteworthy that as the network size grows, due to the asymptotic properties of the power-law graphs the

\footnotetext{
3 http://www-kdd.isti.cnr.it/GERM/

${ }^{4}$ http://odysseas.calit2.uci.edu/doku.php/public:online_social_networks

5 http://www.imdb.com/interfaces
} 
Table 3 Real datasets' summary

\begin{tabular}{|c|c|c|c|c|c|c|c|}
\hline \multirow[t]{2}{*}{ Dataset } & \multirow[t]{2}{*}{ \# Vertices } & \multirow[t]{2}{*}{ \# Edges } & \multirow[t]{2}{*}{ \# Vertex labels } & \multirow[t]{2}{*}{ \# Edge labels } & \multicolumn{3}{|c|}{ Vertex degree } \\
\hline & & & & & Average & Minimum & Maximum \\
\hline Facebook-uniform & 984,830 & 185,508 & 17 & 1 & 0.38 & 0 & 28 \\
\hline Facebook-mhrw & 957,359 & $1,792,188$ & 16 & 1 & 3.74 & 1 & 124 \\
\hline Dblp-9202 & 129,073 & 277,081 & 1 & 11 & 4.29 & 1 & 119 \\
\hline Dblp-0305 & 109,044 & 233,961 & 1 & 3 & 4.29 & 1 & 115 \\
\hline Dblp-0507 & 135,116 & 290,363 & 1 & 3 & 4.28 & 0 & 97 \\
\hline IMDB & $30,835,467$ & $53,686,381$ & 144 & 1 & 1.74 & 1 & 327,915 \\
\hline
\end{tabular}

Table 4 Number of frequent patterns for synthetic data

\begin{tabular}{lllllll}
\hline Network size/pattern size & $10^{2}$ & $10^{3}$ & $10^{4}$ & $10^{5}$ & $10^{6}$ & $10^{7}$ \\
\hline 1 & 3 & 2 & 2 & 2 & 2 & 2 \\
2 & 10 & 4 & 4 & 4 & 4 & 4 \\
3 & 48 & 22 & 22 & 22 & 22 & 22 \\
4 & 295 & 144 & 142 & 142 & 142 & 142 \\
5 & 2,077 & 1,076 & 1,066 & 1,066 & 1,066 & 1,066 \\
6 & 15,698 & 8,605 & 8,534 & 8,534 & 8,534 & \\
7 & 124,687 & 72,084 & 71,688 & 71,688 & & \\
8 & $1,024,216$ & 623,360 & & & & \\
\hline
\end{tabular}

number of frequent patterns of a given size converges. Figure 8 plots for each network size the total time used against the pattern size, for each of the considered algorithms. Note, that we could not run the MINT- BATCH for $10^{7}$ network due to its large memory requirement. For the same reason, for $10^{6}$ network we used batch-size of 20 , while for the rest we set batch-size to 100 .

Sampled frequent patterns of synthetic data The number of patterns grows exponentially. Nevertheless, large patterns may be of interest. A strategy which gained popularity recently (Hasan and Zaki 2009) is to not mine all frequent patterns but only generate a sample of them. Here, we adopt a simple sampling strategy of randomly selecting only 100 frequent patterns at each level (denoted pattern size in our breadth-first mining) of the mining process, to make extensions for the next level. This experiment allows us to study more closely the delay (time used per pattern found) of our miner.

Figure 9 plots for each network the delay (time used per pattern) as a function of the size of the patterns, and also as a function of the size of the network for patterns of size 4 , for each of the considered algorithms.

Real-world datasets Here we followed essentially the same procedure as for synthetic data, the only difference being that we used a smaller frequency threshold of 0.05 for 

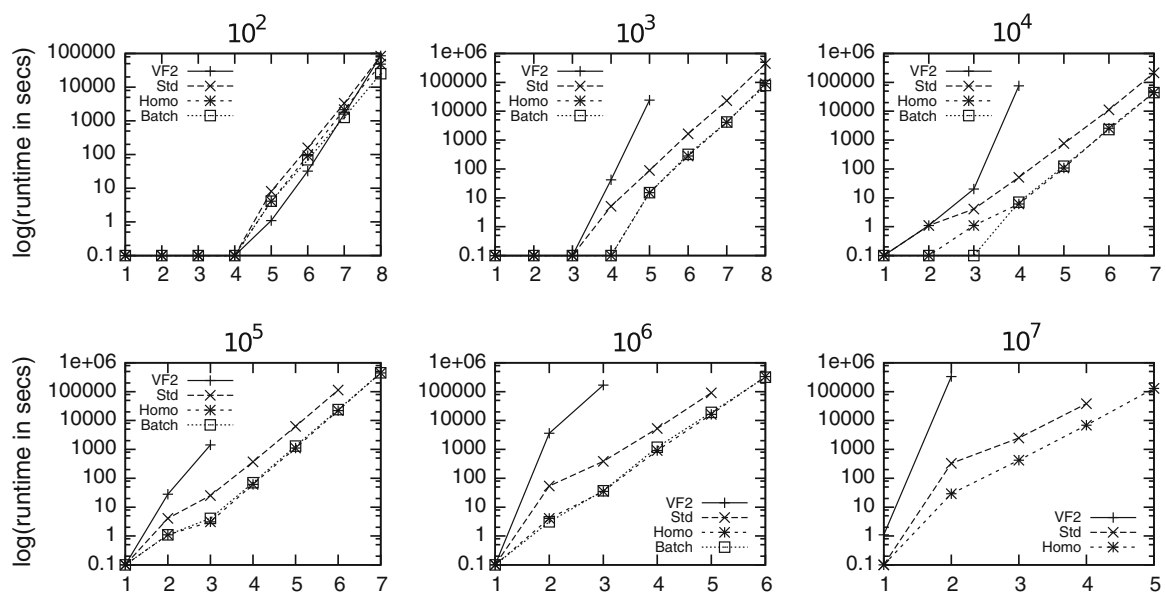

Fig. 8 Log-runtime as a function of pattern size
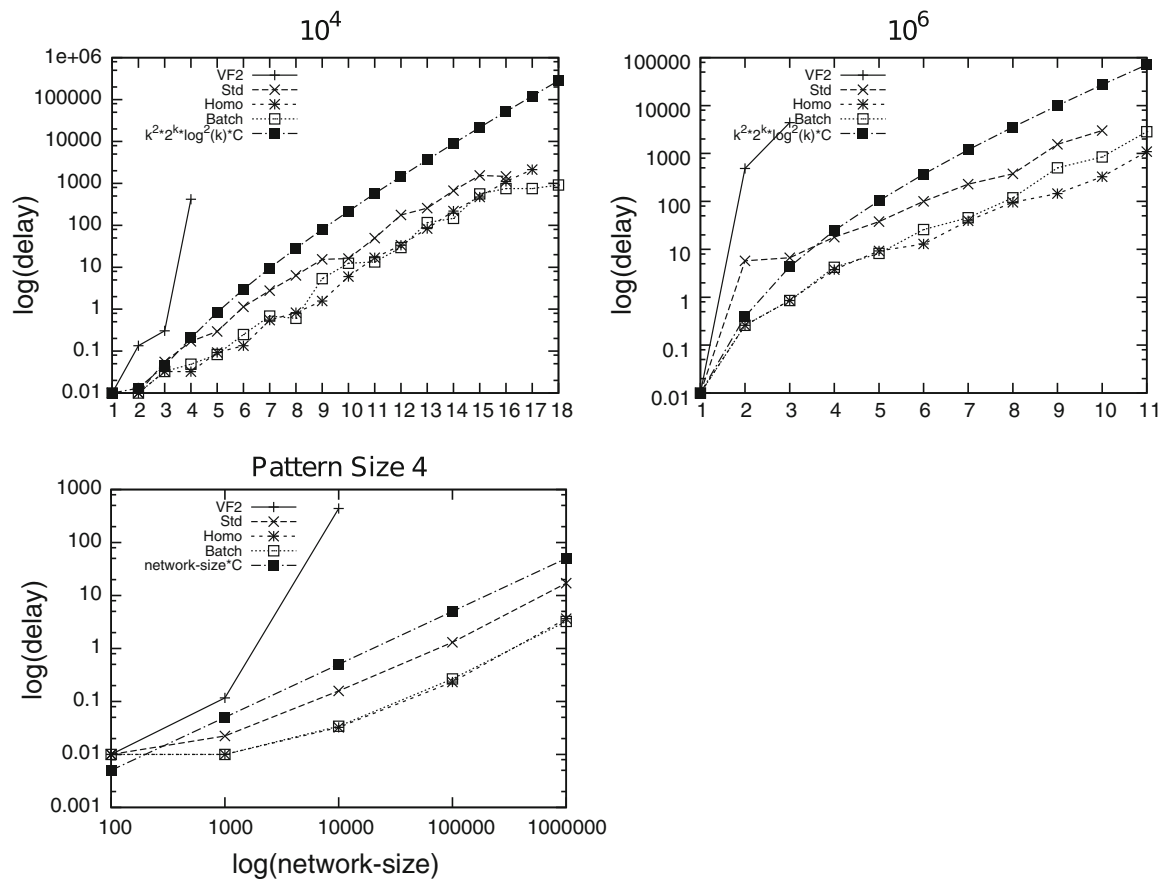

Fig. 9 Log-delaytime (time per pattern) as a function of pattern size, as well as a function of network size

FB-uniform, in order to mine as large patterns as possible. Higher threshold on FBuniform produced very few frequent patterns and we were not able to mine beyond level 5. Like in synthetic data, we used batch-size of 20 for larger networks (Facebook) as parameter for MINT- BATCH, while we could not run it at all on our largest IMDB 
Table 5 Number of frequent patterns for real data

\begin{tabular}{lllllll}
\hline Network/pattern size & FB-uniform & FB-mhrw & Dblp0305 & Dblp0507 & Dblp9202 & IMDB \\
\hline 1 & 2 & 1 & 1 & 1 & 1 & 3 \\
2 & 1 & 2 & 3 & 3 & 8 & 5 \\
3 & 2 & 5 & 12 & 13 & 10 & 10 \\
4 & 3 & 11 & 51 & 57 & 10 & 26 \\
5 & 4 & 30 & 189 & 277 & 6 & 110 \\
6 & 5 & 88 & 648 & 1,099 & 1 & \\
7 & 10 & 299 & 2,140 & 4,459 & 0 & \\
8 & 15 & & & & & \\
\hline
\end{tabular}
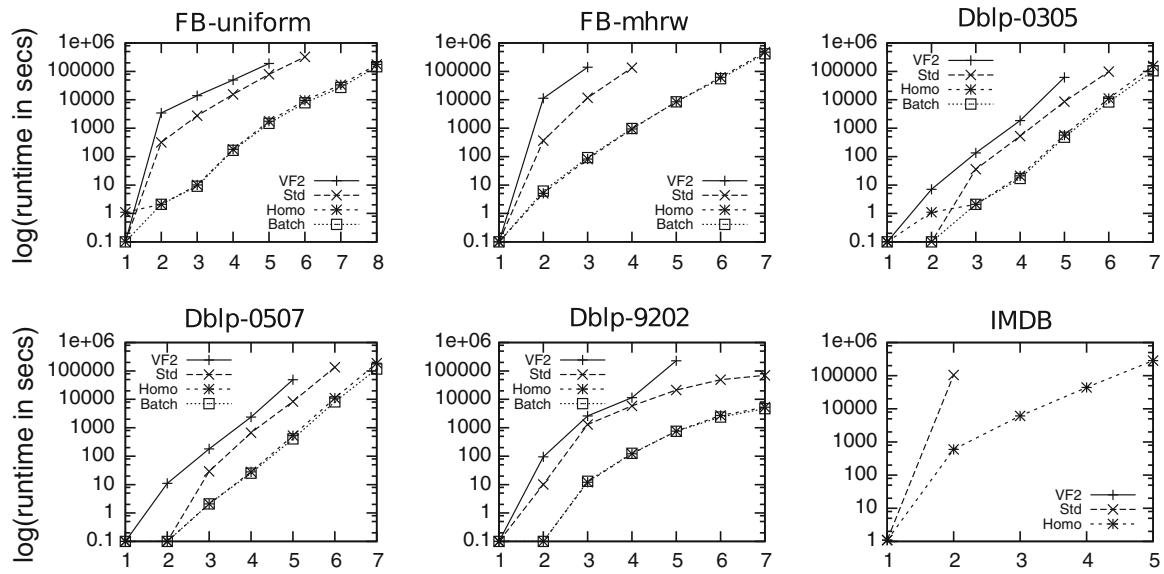

Fig. 10 Log-runtime as a function of pattern size

network. Table 5 lists the number of frequent patterns found for each network. Figure 10 plots for each network the total time used against the pattern size, for each of the considered algorithms. Note that for Dblp-9202 we faced a similar issue as in FBuniform, although less severe. However, we left the frequency threshold there at 0.1 , just to illustrate that if the number of patterns does not grow exponentially, then we are able to mine all frequent trees in a network.

\subsection{Discussion}

Based on the results reported above, we can answer the experimental questions as follows:

Q1 Using the new pattern matching method, it is computationally feasible to match patterns up to size 17-18 (see Fig. 9). We note that for all our experiment settings we mine up to the second last level within $48 \mathrm{~h}$. The main bottleneck when mining all patterns is the number of frequent patterns found. As this number increases exponentially, in many settings we do not get further than pattern size 6-7 for 
larger networks. One can observe however, that for real-world pattern mining tasks one often has prior domain knowledge allowing for pruning the search space towards the type of patterns one is interested in.

Q2 It is clear from all experiments that the new pattern matching method is orders of magnitude better than the VF2 algorithm, especially for larger patterns.

Q3 From Fig. 9 one can see that the pattern matching algorithm scales at least as well as the theoretical upper bound. In particular, in contrast to VF2, our new method scales linearly in the network size and scales indeed as $O\left(k^{2} \log ^{2}(k) 2^{k}\right)$ in the pattern size.

Q4 The homomorphism check prunes away a significant amount of subgraph isomorphism tests for patterns which are clearly infrequent. This especially holds for the real-world dataset.

\section{Potential applications}

In the above experimental evaluation, we have focused on quantitative aspects such as computational efficiency and number of frequent patterns. Another question concerns the meaning of tree patterns in networks. This section aims at providing some potential applications.

\subsection{Using patterns to build predictive models}

Pattern mining is a fundamental step but only a first one. A natural next step is the learning of association rules and/or more complex predictive models. The underlying algorithm for searching and matching predictive patterns could be the same as the one described in this paper and could have several uses. First, rule-based predictive models such as association rules or decision trees in networks need an algorithm to search patterns correlating with the target variable. For instance, rules could be of the form

If pattern $P$ can be embedded in the network such that root $r$ is mapped to $x$, then $x$ has label $l$.

Second, embeddings of patterns could serve as templates for observations or relationships of a particular form. For instance, probabilistic logical/relational models (Getoor and Taskar 2007) contain conditional probability distributions between random variables. Several of these models allow to specify patterns such that all embeddings of the pattern correspond to a dependency. For instance, the value of a painting $x$ may depend on the amount in the savings accounts $a$ of all art collectors $c$ admiring the painter $p$ who painted $x$. A conditional probability distribution could hence specify that the value of the painting $x$ is gaussian distributed around the maximum of the amount labels associated to the account nodes in the set of all embeddings of the path $P$ with $V(P)=\{x, p, c, a\}$ and $E(P)=\{\{x, p\},\{p, c\},\{c, a\}\}$. For instance, in the Logical Bayesian Network model (Fierens et al. 2005) this would be represented with

$$
\operatorname{value}(x) \mid \operatorname{amount}(a) \leftarrow \operatorname{painted}(p, x), \operatorname{admires}(c, p), \operatorname{account}(a, c)
$$




\subsection{Some more real-world examples}

In many real-world cases, patterns of interest are tree shaped. Let us consider a few more examples. A typical network application is a social network. A possible rule involving a tree-shaped pattern is

If person $X$ has a friend $Y$ and $Y$ has posted a message $M$ and this message $M$ mentions football and $X$ lives in state $C$ and $C$ is in North-America, then $X$ may be interested in American football.

In social networks often closely related nodes have most influence on properties of a node. In administrative applications, however, administrative constructions may often extend over several nodes as some legal rules should be followed which can mention several objects between which certain administrative relations hold. For instance, imagine a network consisting of company ownership and family/kinship relations. In many countries there are laws concerning ownership or directorship of new businesses while an existing business is in liquidation or has filed for bankruptcy. One can imagine that there would be individuals who would want to circumvent regulations by starting or controlling other businesses through proxy. A rule of interest could be

If a company $C 1$ went bankrupt and person $P 1$ is the owner of $C 1$, and $P 1$ has a likely proxy (e.g. family member) $P 2$ and $P 2$ started a company $C 2$, then the probability that $C 2$ will go bankrupt is higher.

We can detect such potential violation of regulations by mining rooted tree patterns, performing a guided search by growing patterns using "bankrupt" as the seed/root and mining patterns similar to the one above. As we showed in the experiments, it is possible to mine patterns significantly larger than the examples above and so reasonably complex situations can be modeled.

Of course, not all patterns of importance will be tree-shaped. The work in this paper is a first important step but in future work it would be definitely of interest to be able to also handle non-tree patterns. However, to the best of our knowledge, for matching non-tree patterns no algorithm is known with linear running time in the size of the network.

\section{Conclusion and future work}

We present a novel algorithm for mining trees in single networks. It scales well with respect to network size, and is only mildly exponential in pattern size, which makes it tractable for moderately sized patterns. Our method works for both sparse and dense graphs. We show the effectiveness of the method in practice, on real as well as synthetic data.

Our method is based on recent results from fixed parameter tractability research. Indeed, in pattern mining tasks in large networks we typically face pattern sizes which are orders of magnitude smaller than the networks which may contain millions to billions of nodes. In such cases, algorithms that are tractable in the largest parameter, in our case even linear in the network size, are preferable. We also believe more 
generally that a better understanding of parameterized complexity can be helpful in overcoming the computational challenges of data mining.

There are several interesting lines of future work. First, we pointed out that the combination of our method with sampling techniques may be nontrivial. Further research to develop a sampling-friendly algorithm would be a useful next step. Second, while our work concerns tree patterns, we believe that it is possible to extend our methods to more general graph classes. Third, our approach is currently an in-memory approach. For really huge databases, further improvements are needed to let our algorithm organize data access economically. Fourth, sometimes one may be interested in even larger patterns (e.g. of more than 30 vertices). It is not straightforward to develop an algorithm to mine large patterns, and possibly mining large patterns is also a computationally untractable problem. On the other hand, it is also possible that for practical applications the worst case never happens and a good strategy can be found. Finally, this article focuses on pattern matching. However, when mining large networks, we also need to pay attention to the support measure, taking into account the dependencies between our observations (embeddings) rather than just counting their number. We intend to generalize our implementation such that it can be effectively used to compute also other support measures.

Acknowledgements This research is supported by ERC Starting Grant 240186 "MiGraNT: Mining Graphs and Networks, a Theory-Based Approach". We thank Anton Dries and Constantin Commendant for the valuable suggestions.

\section{References}

Berlingerio M, Bonchi F, Bringmann B, Gionis A (2009) Mining graph evolution rules. In: Proceedings of the European conference on machine learning and knowledge discovery in databases: part I, ECMLPKDD '09. Springer, Berlin, pp 115-130

Blum M, Kannan S (1995) Designing programs that check their work. J ACM 42(1):269-291

Bogdanov P, Mongiovì M, Singh AK (2011) Mining heavy subgraphs in time-evolving networks. In: Proceedings of the (2011) IEEE 11 th international conference on data mining, ICDM '11. IEEE Computer Society, Washington, DC, pp 81-90

Borgelt C, Berthold MR (2002) Mining molecular fragments: finding relevant substructures of molecules. In: Proceedings of the (2002) IEEE international conference on data mining, ICDM '02. IEEE Computer Society, Washington, DC, pp 51-58

Borgelt C, Meinl T, Berthold M (2005) Moss: a program for molecular substructure mining. In: Proceedings of the 1st international workshop on open source data mining: frequent pattern mining implementations, OSDM '05. ACM, New York, pp 6-15

Bringmann B, Nijssen S (2007) What is frequent in a single graph? In: Frasconi P, Kersting K, Wrobel S (eds) Proceedings of MLG-2007: 5th international workshop on mining and learning with graphs, pp $1-4$

Calders T, Ramon J, Van Dyck D (2011) All normalized anti-monotonic overlap graph measures are bounded. Data Min Knowl Discov 23(3):503-548

Chehreghani M (2011) Efficiently mining unordered trees. In: 2011 IEEE 11th international conference on data mining (ICDM), pp 111-120

Chen J, Kanj IA, Jia W (2001) Vertex cover: further observations and further improvements. J Algorithm 41(2):280-301

Chi Y, Yang Y, Muntz R (2004) Hybridtreeminer: an efficient algorithm for mining frequent rooted trees and free trees using canonical forms. In: Proceedings of 16th international conference on scientific and statistical database management, 2004, pp 11-20 
Chi Y, Xia Y, Yang YR, Muntz R (2005) Mining closed and maximal frequent subtrees from databases of labeled rooted trees. IEEE Trans Knowl Data Eng 17:190-202

Cook DJ, Holder LB (1994) Substructure discovery using minimum description length and background knowledge. J Artif Intell Res 1(1):231-255

Cook DJ, Holder LB (2000) Graph-based data mining. IEEE Intell Syst 15(2):32-41

Cordella LP, Foggia P, Sansone C, Vento M (2001) An improved algorithm for matching large graphs. In: 3rd IAPR-TC15 workshop on graph-based representations in pattern recognition, Cuen, pp 149-159

Cordella LP, Foggia P, Sansone C, Vento M (2004) A (sub)graph isomorphism algorithm for matching large graphs. IEEE Trans Pattern Anal Mach Intell 26:1367-1372

Diestel R (2010) Graph theory, 4th edn, electronic edn. Springer, New York

Dries A, Nijssen S (2012) Mining patterns in networks using homomorphism. In: Proceedings of the twelfth SIAM international conference on data mining, pp 260-271. Omnipress, Madison. https://lirias. kuleuven.be/handle/123456789/350328

Fierens D, Blockeel H, Bruynooghe M, Ramon J (2005) Logical Bayesian networks and their relation to other probabilistic logical models. In: Proceedings of the 15th international conference on inductive logic programming. Lecture notes in computer science, vol 3625. Springer, New York, pp 121-135

Gallagher B, Tong H, Eliassi-Rad T, Faloutsos C (2008) Using ghost edges for classification in sparsely labeled networks. In: Proceedings of the 14th ACM SIGKDD international conference on Knowledge discovery and data mining, KDD '08. ACM, New York, pp 256-264

Getoor L, Taskar B (2007) An Introduction to statistical relational learning. MIT Press, Cambridge

Gjoka M, Kurant M, Butts C, Markopoulou A (2010) Walking in facebook: a case study of unbiased sampling of OSNs. In: Proceedings of IEEE INFOCOM '10

Hasan MA, Zaki MJ (2009) Output space sampling for graph patterns. Proc VLDB Endow 2(1):730-741

Henderson K, Gallagher B, Li L, Akoglu L, Eliassi-Rad T, Tong H, Faloutsos C (2011) It's who you know: graph mining using recursive structural features. In: Proceedings of the 17th ACM SIGKDD international conference on Knowledge discovery and data mining, KDD '11. ACM, New York, pp 663-671

Horváth T, Ramon J (2010) Efficient frequent connected subgraph mining in graphs of bounded tree-width. Theor Comput Sci 411:2784-2797

Horváth T, Ramon J, Wrobel S (2010) Frequent subgraph mining in outerplanar graphs. Knowl Discov Data Min 21(3):472-508

Huan J, Wang W, Prins J (2003) Efficient mining of frequent subgraphs in the presence of isomorphism. In: Proceedings of the (2003) third IEEE international conference on data mining, ICDM '03. IEEE Computer Society, Washington, DC, pp 549-556

Huan J, Wang W, Prins J, Yang J (2004) Spin: mining maximal frequent subgraphs from graph databases. In: Proceedings of the 10th ACM SIGKDD international conference on Knowledge discovery and data mining, KDD '04. ACM, New York, pp 581-586

Inokuchi A, Washio T, Motoda H (2000) An apriori-based algorithm for mining frequent substructures from graph data. In: Proceedings of the 4th European conference on principles of data mining and knowledge discovery, PKDD '00. Springer, London, pp 13-23

Jiang X, Xiong H, Wang C, Tan AH (2009) Mining globally distributed frequent subgraphs in a single labeled graph. Data Knowl Eng 68(10):1034-1058

Kibriya AM, Ramon J (2012) Nearly exact mining of frequent trees in large networks. Proceedings of the (2012) European conference on machine learning and knowledge discovery in databases-volume part I, ECML PKDD'12. Springer, Berlin, pp 426-441

Koutis I (2008) Faster algebraic algorithms for path and packing problems. In: Proceedings of ICALP '08. Springer, New York, pp 575-586

Koutis I, Williams R (2009) Limits and applications of group algebras for parameterized problems. In: Proceedings of ICALP '09. Springer, New York, pp 653-664

Kuramochi M, Karypis G (2001) Frequent subgraph discovery. In: Proceedings of the (2001) IEEE international conference on data mining, ICDM '01. IEEE Computer Society, Washington, DC, pp 313-320

Kuramochi M, Karypis G (2005) Finding frequent patterns in a large sparse graph*. Data Min Knowl Discov 11(3):243-271

Motwani R, Raghavan P (1995) Randomized algorithms. Cambridge University Press, New York

Nienhuys-Cheng SH, De Wolf R (1997) Foundations of inductive logic programming. Lecture notes in computer science and lecture notes in artificial intelligence, vol 1228. Springer, New York

Nijssen S, Kok J There is no optimal, theta-subsumption based refinement operator. Personal communication 
Nijssen S, Kok JN (2004) A quickstart in frequent structure mining can make a difference. In: Proceedings of the 10th ACM SIGKDD international conference on knowledge discovery and data mining, KDD '04. ACM, New York, pp 647-652

Nijssen S, Kok JN (2005) The gaston tool for frequent subgraph mining. Electron Notes Theor Comput Sci 127(1):77-87; Proceedings of the international workshop on graph-based tools (GraBaTs 2004)

Robson JM (1986) Algorithms for maximum independent sets. J Algorithm 7(3):425-440

Rossi RA, McDowell LK, Aha DW, Neville J (2012) Transforming graph data for statistical relational learning. J Artif Intell Res 45:363-441

Thomas LT, Valluri SR, Karlapalem K (2010) Margin: maximal frequent subgraph mining. ACM Trans Knowl Discov Data 4:10:1-10:42

Ullmann J (1976) An algorithm for subgraph isomorphism. J Assoc Comput Mach 23(1):31-42

Wang Y, Ramon J, (2012) An efficiently computable support measure for frequent subgraph pattern mining. In: Proceedings of ECML/PKDD, (2012) Lecture notes in computer science, vol 7523. Springe, New York, pp 362-379

Wörlein M, Meinl T, Fischer I, Philippsen M (2005) A quantitative comparison of the subgraph miners mofa, gspan, ffsm, and gaston. Proceedings of the 9th European conference on principles and practice of knowledge discovery in databases, PKDD '05. Springer, Berlin, pp 392-403

Yan X, Han J (2002) gspan: Graph-based substructure pattern mining. In: Proceedings of the (2002) IEEE international conference on data mining, ICDM '02. IEEE Computer Society, Washington, DC, pp $721-724$

Yan X, Han J (2003) Closegraph: mining closed frequent graph patterns. In: Proceedings of the 9th ACM SIGKDD international conference on knowledge discovery and data mining, KDD '03. ACM, New York, pp 286-295 